\title{
Dislocation After Bipolar Hemiarthroplasty in Elderly Patient: A Case Report
}

\author{
Cokorda Gde Oka Dharmayuda, and Febyan
}

\section{ABSTRACT}

Total hip arthroplasty and hemiarthroplasty are two primary treatment choices for elderly patients with fractures or dislocations of the pelvic bone, as well as in fractures of the femoral neck. Dislocation of bipolar hemiarthroplasty is a rare postsurgical complication, but it can be a problem that requires acute management and is quite challenging to revise. We presented a case of an elderly patient with dislocation of hip bipolar hemiarthroplasty on the right side. The patient underwent a revision of total hip arthroplasty and had a satisfactory outcome. Mobilization began within two days after surgery. One-year follow-up revealed no occurrence of longterm complications.

Keywords: Bipolar hemiarthroplasty, dislocation, elderly patient, total hip arthroplasty.
Submitted: October 20, 2021

Published: November 13, 2021

ISSN: 2593-8339

DOI: $10.24018 /$ ejmed.2021.3.6.1112

\section{G. O. Dharmayuda*}

Department of Orthopaedic and Traumatology, Faculty of Medicine, Udayana University, Sanglah Public General Hospital, Denpasar, Bali, Indonesia.

(e-mail: dharmayudadr@gmail.com) Febyan

Department of Orthopaedic and Traumatology, Bhayangkara Denpasar Hospital, Bali, Indonesia.

(e-mail: febyanmd@gmail.com)

*Corresponding Author

\section{INTRODUCTION}

Bipolar hemiarthroplasty (BHA) is one of the surgical treatment choices for unstable fractures of the femoral neck in elderly patients, or as a secondary procedure after a failed attempt of internal fixation [1]. This procedure is advantageous because it provides an immediate full weightbearing and a decreased reoperation rate, which subsequently reduces postoperative morbidity rates [2]. Hip dislocation after BHA prosthesis is rare but is a serious complication requiring an urgent open reduction and revision. The incidence of dislocation is not significantly different between unipolar and bipolar hemiarthroplasty [3]. Our study reported a case of the dislocated hip following the BHA surgery. Informed consent was obtained from the patient for publication of this case report and images for medical education purposes.

\section{Presentation of CASe}

A 70-year-old female patient presented to the emergency department with a chief complaint of right hip pain and a history of fall that occurred four months ago. Six months prior to the current visit, the patient had a history of right-sided femoral neck fracture and had undergone bipolar hemiarthroplasty surgery. Physical examination revealed shortening and external rotation on the affected limb, indicating hip dislocation. The patient also suffered pain on palpation and presented with a limited range of motion (active and passive movement). Laboratory tests revealed no abnormal findings. A plain radiograph was taken and demonstrated findings consistent with dislocation of the femoral head (Fig. 1). We informed the patient to be surgically treated by an open reduction followed by total hip arthroplasty (THA) and gained consent to perform the surgery. Revisional surgery was performed through the posterior approach. The femur was gathered anteriorly, followed by removing the existing bipolar hip arthroplasty implant. Ensure that the fibrous tissue had been removed thoroughly so that the acetabulum could be identified. Using an acetabular reamer, gently ream onto the remaining acetabular bone and place the implant within the acetabulum. The implant was later cemented onto proper positioning. Place the cerclage wire for the femoral bone and ream. Insert the long cemented femoral stem, and then the surgery was completed by reducing the hip. Subsequently, the patient was alert and presented with no further complication on postoperative radiograph follow-up (Fig. 2). Mobilization began within two days after surgery. We also recommended the patient undergo rehabilitation protocol. At one year of follow-up, the patient had no complaints and was able to carry out normal daily activities without any pain. 


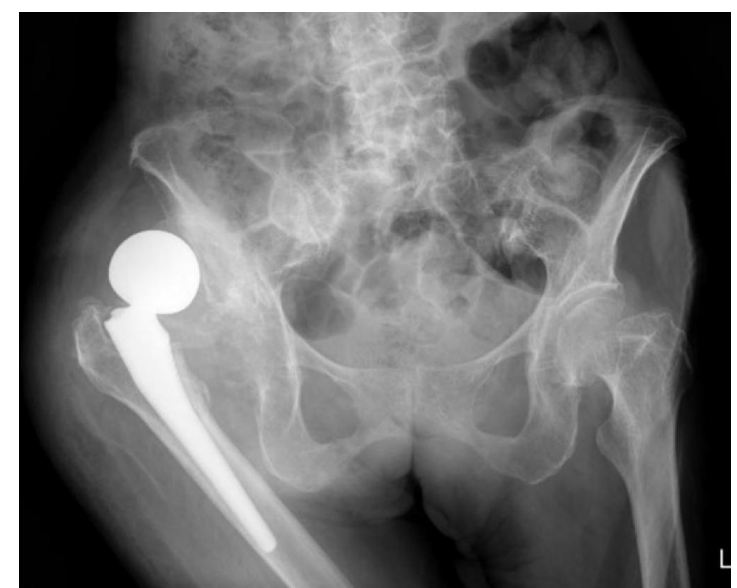

Fig. 1. The anteroposterior radiograph showed hip dislocation with bipolar hemiarthroplasty on the right side.

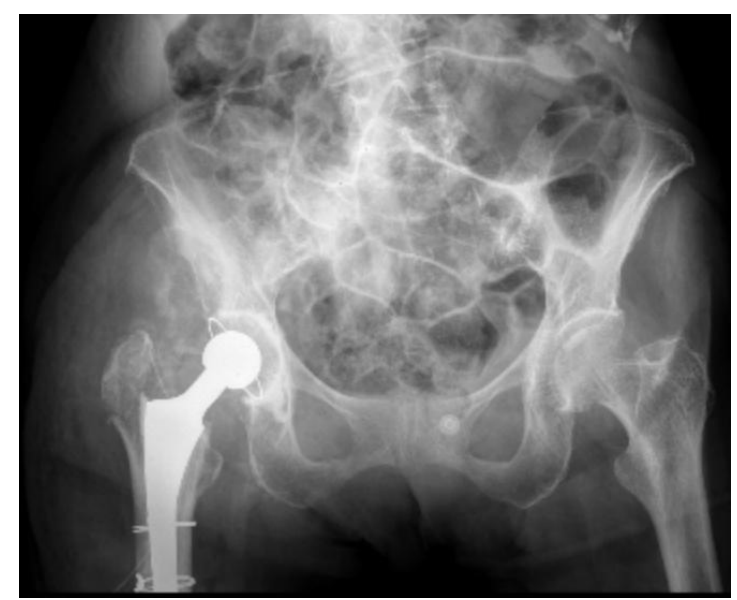

Fig. 2. The postoperative radiograph following the open reduction and total hip arthroplasty was fixed by wire placement.

\section{Discussion}

Total hip arthroplasty (THA) and hemiarthroplasty are two primary treatment choices for elderly patients with fractures or dislocations of the pelvic bone, as well as in fractures of the femoral neck [4]. Our patient had a history of right-sided femoral neck fracture and had undergone hip BHA six months before the current visit. The patient presented with dislocation after the initial surgery, in which the femoral head component was entirely out of the acetabulum and had migrated proximally. Dislocation of the prosthetic cup and femoral head following BHA is a rare complication; however, it may lead to serious complications requiring an acute revision procedure, which is quite challenging for the surgeons [5].

A study by Philips et al. reported the complications in the first six months postoperatively and found an overall dislocation rate of $3.9 \%$, with $3 \%$ occurring in the first eight weeks after surgery [6]. Previous studies have stated that the incidence of dislocation is related to the small acetabular depth and small acetabular depth-width ratio. The elderly patients with a center-edge angle of $\leq 45.4^{\circ}(p<0.001)$ or an acetabular depth of $\leq 19.12 \mathrm{~mm}(\mathrm{p}<0.001)$ were susceptible to experience dislocation after BHA [7]. A comprehensive review by Jones et al. explained several factors related to HA dislocation, including patient cognition, preceding surgery failure, delay to surgery, types of surgical approach, and the femoral offset [8]. Our patient also had a history of falling after surgery; thus, it became a traumatic factor that causes a direct force to the hip HA site.

The challenge of our case was the presence of fibrosis formation at the acetabulum surface, which occurred within four months throughout the dislocation. We cleared out the fibrosis formation and replaced the natural acetabulum with metal. Moriarity et al. [9] stated that the dislocated hip HA should be managed by open reduction and be converted to cemented THA. This study was supported by Fan et al., who performed revision surgery of BHA to THA with dual mobility cups, which showed an excellent postoperative result [10].

A comprehensive inspection of the acetabulum and adjacent structures for any defects or fractures should be performed, followed by examining the femoral stem for loosening and correct version. In our case, the patient had a well-fixed femoral stem with good alignment. We only prepared the Hastings femoral head component for the complete revision of the hemiarthroplasty components to prevent further dislocations.

\section{CONCLUSION}

Surgical hip hemiarthroplasty in active elderly patients, especially those with a shallow acetabulum, is vulnerable to complications of hemiarthroplasty dislocation. The total hip arthroplasty should be considered to gain an excellent longterm surgical outcome and prevent further complications, including recurrence of dislocation.

\section{REFERENCES}

[1] Enocson A., Tidermark J., Tornkvist H., Lapidus LJ., Dislocation of hemiarthroplasty after femoral neck fracture. Acta Orthopaedice. 2008; 79(2): 211-7.

[2] Gjertsen JE., Vinje T., Engesaeter LB., Lie SA., Havelin LI., Furnes O., et al. Internal screw fixation compared with bipolar hemiarthroplasty for treatment of displaced femoral neck fractures in elderly patients. J Bone Joint Surg Am. 2010; 92: 619-28.

[3] Madanat R., Makinen TJ., Ovaska MT., Soiva M., Vahlberh T., Haapala J. Dislocation of hip hemiarthroplasty following posterolateral surgical approach: a nested case-control study. Int Orthop. 2012; 36: 935-40.

[4] Zhao Y., Fu D., Chen K., Li G., Cai Z., Shi Y., et al, Outcome of hemiarthroplasty and total hip replacement for active elderly patients with displaced femoral neck fractures: a meta-analysis of 8 randomized clinical trials. PLOS ONE. 2014; 9(5): 1-7.

[5] Mathews CS., Garrison RL., Renard RL. Use of a fracture table for irreducible bipolar hemiarthroplasty dislocation: a case report. Int $J$ Case Rep Images. 2017; 8(2): 138-42.

[6] Philips CB., Barrett JA., Losina E., Lingard EA., Guadagnoli E., Baron JA., et al. Incidence rates of diclocation, pulmonary embolism, and deep infection during the first 2003; 85: 20.

[7] Zhang Y., Yao Z., Shi P., Wang C., Liu J., Yang Y., et al. Morphological risk factors associated with dislocation after bipolar hemiarthroplasty of the hip in patients with femoral neck frcatures- a nested case control study. Journal of Orthopaedics Surgery and Research 2019; 1-7.

[8] Jones C., Briffa N., Jacob J., Hargrove R. The dislocated hip hemiarthroplasty: current concepts of etiological factors and management. The Open Orthopaedics Journal 2017; 11(7): 1200-12.

[9] Moriarity A., Ellanti P., Talha S., McKenna J. Dislocation and dissociation of bipolar hip hemiarthroplasty. BMJ Case Rep. 2015;1-3.

[10] Fan XL., Wang J., Zhang D., Mao F., Liao Y. Early reccurent dislocation and dissociation of bipolar cup: two intricate cases and review of literature. Research Square 2020; 1-12. 